\title{
LA RAPPRESENTAZIONE MEDIATICA DEL MIGRANTE TRA ACCOGLIENZA E DIFFIDENZA
}

\author{
Snježana Bralić, Filozofskifakultet Sveučilišta u Splitu, sbralic@ffst.hr \\ Maja Bezić, Filozofski fakultet Sveučilišta u Splitu mbezic@ffst.hr
}

\subsection{2/fIl.30.2020.17 \\ UDK 314:316.77(450)}

Negli ultimi decenni del Novecento le nuove guerre, le pulizie etniche e i disastri ambientali hanno creato un alto numero di migranti e profughi, persone in fuga e in transito che si spostano alla ricerca di migliori condizioni di vita. Dato che l'Europa, e in particolar modo l'Italia, si sentono in pericolo, colpiti dalla sindrome d'invasione per i continui arrivi di immigrati, si sono formati nuovi muri, non solo materiali, ma anche muri e frontiere mentali. Il nuovo clima ha favorito la nascita di parole nuove relative ai movimenti migratori e alla percezione della figura del migrante. Con la crescita del fenomeno, si è diffusa un'epidemia di pregiudizi e stereotipi di fronte alle persone percepite come oggettivamente diverse. I termini e le espressioni, a cui si ricorre per indicare i nuovi arrivati, abbondano di etichette del migrante la cui rappresentazione mediatica risulta per lo più negativa.

Da diversi studi che trattano questo argomento si percepisce che il discorso mediatico italiano, centrato sull'emergenza, contribuisce alla stereotipizzazione negativa dello straniero, legata alla criminalità e pericolosità. Secondo Maneri e Dal Lago lo straniero viene percepito come una minaccia alla società italiana ed europea e discriminato innanzitutto dal linguaggio usato per rappresentarlo, mentre la contrapposizione tra Noi e Loro viene rafforzata da generalizzazioni e dall'uso del lessico metaforico.

Il corpus che si propone di studiare e analizzare si riferisce agli articoli sul tema delle migrazioni, tratti dai due giornali quotidiani italiani di diffusione nazionale. Si prendono in esame gli articoli pubblicati in due periodi diversi, corrispondenti ai differenti contesti sociopolitici della realtà italiana (settembre 2015 e aprile 2017), e in tal modo si tenta di osservare e studiare la lingua nel suo ruolo da protagonista nella costruzione dell'immagine mediatica dei migranti.

Parole chiave: fenomeno delle migrazioni, discorso mediatico italiano, strategie discorsive, stereotipizzazione negativa dello straniero, accoglienza e diffidenza 


\section{Introduzione}

Nella seconda metà degli anni Settanta I'Italia subisce la trasformazione da un paese degli emigrati in un paese degli immigrati. Questa trasformazione si accelera negli anni Novanta, mentre nel primo decennio del XXI secolo I'Italia diventa uno dei paesi più ambiti da parte degli immigrati (Triandafyllidou 1999, Sciortino, Colombo 2004, Taylor 2009).

Da quando I'Italia è diventata paese d'immigrazione, lo spostarsi e il migrare hanno dato il proprio contributo al lessico italiano che nel corso degli ultimi decenni ha sostituito i termini e le espressioni inizialmente offensivi e degradanti con un modo di comunicare ufficiale. Riferendosi alle parole di qualche decennio indietro, quando gli immigrati erano ancora pochi, venivano etichettati come vu' cumprà, lavavetri, marocchini, mentre nel linguaggio burocratico sono stati creati i termini come extracomunitario, irregolare o clandestino. Da una parte con il termine vu' cumprà si indicavano i nuovi arrivati chiamati in questo modo per lo stentato italiano con cui offrivano la loro mercanzia e dall'altra parte il termine marocchino veniva usato per tutti i magrebini e talvolta anche per tutti gli africani (Sciortino, Colombo 2004).

Intorno alla metà degli anni Novanta, la situazione cambia rapidamente e i nomi per chi viene da fuori si moltiplicano. Alcuni, come extracomunitario, si formano nel linguaggio burocratico per indicare gli stranieri che non fanno parte dell'Unione Europea, ma utilizzato quasi esclusivamente a proposito degli immigrati abbandonati e in condizioni di vita piuttosto misere. Da allora, nell'opinione comune, la condizione di extra-comunitarietà viene sempre più spesso legata a quella di chi delinque, senza la verifica se questo corrisponda a verità. C'è inoltre la tendenza di considerare tutti gli immigrati come degli irregolari (cioè con i documenti ma senza permesso di soggiorno) oppure come dei clandestini (senza documenti e senza permesso). Quindi, in quel periodo degli anni Novanta l'immigrato viene spesso sentito come un alieno, lo straniero in generale come un pericolo, come un potenziale delinquente.

Nel contempo i termini e le espressioni più offensivi e degradanti sono stati sostituiti, soprattutto negli ambiti ufficiali. Così per indicare uno straniero non in regola con la legislazione si preferiva usare il termine francese sans papier ('senza documenti'), mentre dall'altra parte complessa e lunga è stata la strada per cui da un negro si è arrivati a uomo o donna di colore o all'altrettanto eufemistica espressione non bianco (Faloppa 2011). II problema e il rischio, in generale, sta nel fatto che gli extracomunitari, immigrati o migranti 
nelle società occidentali rimangano dei senzanome, dei soggetti caratterizzati da uno stato di invisibilità civile. Anche se le società occidentali come d'altronde anche quella italiana sono già società multiculturali e multietniche, non sembrano pronte al multiculturalismo e alla multiculturalità, a una coabitazione con lo straniero dove prevalga una cultura dell'accoglienza che favorisca la convivenza delle differenze.

\section{Panoramica degli studi sul fenomeno migratorio}

Parlando del tema delle migrazioni, che continua a suscitare sempre maggior interesse non soltanto da parte dei media ma anche degli studiosi di varie discipline, bisogna menzionare diversi rapporti nati nell'ambito dei progetti incentrati sull'analisi della rappresentazione mediatica dei migranti e numerosi studi sull'immigrazione pubblicati nell'arco degli ultimi trent'anni.

Tra gli studi sul tema delle migrazioni, spiccano gli studi sociologici e antropologici, mentre molto meno numerosi sono gli studi che trattano il ruolo della lingua nella raffigurazione dei migranti. $\grave{E}$ doveroso menzionare Alessandro Dal Lago, noto sociologo italiano, autore di Non-persone. L'esclusione dei migranti in una società globale (1999), uno dei libri fondamentali negli studi sulla condizione dei migranti in Italia. Nel libro Dal Lago studia diversi ambiti dell'esclusione e della segregazione dei migranti nella società parlando "di una simbologia che trasforma la distinzione puramente empirica tra noi e loro in una contrapposizione ontologica, ciò̀ tra mondi radicalmente opposti. Prima ancora di essere discriminati nei fatti, migranti $e$ profughi sono discriminati dal linguaggio che la nostra società escogita per rappresentarli." (Dal Lago 1999, 43)

Secondo Marcello Maneri (1998), un altro famoso sociologo italiano, alla fine degli anni Ottanta, all'interno di una rappresentazione mediatica generalmente negativa e spesso allarmistica, si nota il primo cambiamento ovvero il passaggio dall'emergenza razzismo all'emergenza criminalità, mentre la contrapposizione tra Noi e Loro viene rafforzata da generalizzazioni e dall'uso del lessico, spesso metaforico, relativo al campo semantico della guerra e a quello dell'acqua.

Quanto all'impiego della lingua usata nella raffigurazione dei migranti, evocato da Dal Lago e Maneri, vanno menzionati gli studi di stampo linguistico, tra i quali vengono individuati gli studi di Jessica ter Wal (2000, 2001, 2002), Federico Faloppa (2000, 2004, 2011), Charlotte Taylor (2009, 2014), Lorenzo Montali, Paolo Riva, Alessandra Frigerio e Silvia Mele (2013) e Paolo Orrù (2017). Partendo dai risultati di vari tipi 
di analisi quantitative e qualitative, condotte negli studi citati, si arriva alla stessa conclusione: il discorso mediatico italiano, centrato sull'emergenza, contribuisce alla stereotipizzazione negativa dello straniero, legata alla criminalità e pericolosità, mentre i migranti vengono percepiti come una minaccia alla società italiana ed europea in generale.

\section{Impostazioni teoriche}

A partire dal quadro teorico della ricerca impostato sull'analisi critica del discorso (CDA) (Reisigl e Wodak 2001) e servendosi delle fonti giornalistiche, questo intervento si propone di analizzare le strategie referenziali e le strategie predicative usate nella rappresentazione dei migranti nei quotidiani italiani. Si cerca di rispondere alla domanda come vengono denominati e raffigurati $i$ migranti nel discorso mediatico italiano. Si osserva l'impiego di riferimenti generici, riferimenti collettivi, quantificazione, qualificazione attributiva e sintagmi relativi al fenomeno migratorio.

Le strategie referenziali e le strategie predicative fanno parte delle strategie discorsive usate nella rappresentazione positiva di sé e quella negativa dell'altro. Si riferiscono al modo in cui vengono denominati e qualificati gli attori sociali, spesso evocando gli stereotipi negativi e le connotazioni degradanti e offensive nei riguardi dell'altro percepito come oggettivamente diverso. Tra le diverse strategie referenziali proposte da Reisigl e Wodak (2001), in questa sede si analizzano la genericizzazione, la collettivizzazione, l'aggregazione e l'impiego dei sintagmi relativi al fenomeno migratorio. L'aggregazione comprende la quantificazione attraverso la quale gli attori sociali vengono percepiti come numeri e statistiche e perciò spersonalizzati. D'altra parte, tramite l'impiego dei riferimenti generici e di quelli collettivi, gli attori sociali vengono raffigurati in modo generico, come un gruppo (ad es. i migranti, gli immigrati, gli stranieri), oppure in modo collettivo, come un'entità (ad es. popolo, nazione, popolazione). Con lo scopo di comprendere meglio il contesto nel quale questi riferimenti vengono usati e le connotazioni che essi veicolano, vengono studiati gli attributi che li accompagnano e che qualificano il referente assegnandogli certe caratteristiche o qualità. Essi appartengono alla qualificazione attributiva la quale fa parte delle strategie predicative. Va notato che le strategie predicative non possono essere nettamente distinte dalle strategie referenziali in quanto alcune strategie referenziali, oltre a nominare gli attori sociali, evocano le loro qualità positive o negative influendo in tal modo sulla formazione dell'opinione pubblica (Reisigl e Wodak 2001). 


\section{Parole per definire o per etichettare lo straniero?}

\subsection{Corpus}

Il corpus abbraccia gli articoli sul tema delle migrazioni tratti dalle rubriche di attualità delle edizioni online di due quotidiani nazionali: la Repubblica ${ }^{1}$, quotidiano generalista, e il Giornale ${ }^{2}$, quotidiano di orientamento politico centro-destra. Nonostante gli orientamenti diversi dei due giornali prescelti che contribuiscono a una raffigurazione del fenomeno migratorio, meno oppure più sensazionalistica, da una parte, e meno oppure più politicamente corretta, dall'altra, in questo studio si cerca di analizzare le comuni chiavi interpretative di questa nuova realtà italiana, fornite dai mass media. Con lo scopo di fare un'analisi sia sincronica che diacronica, vengono presi in esame gli articoli pubblicati in due periodi diversi, nel corso di due settimane: dal 14 al 30 settembre 2015 (158 articoli, 99634 parole) e dal 14 al 30 aprile 2017 (91 articoli, 47273 parole). Vale a dire che i due periodi osservati corrispondono ai particolari momenti della realtà sociale e politica italiana, ovvero ai contesti differenti nell'ambito dei quali viene studiata la lingua nel suo ruolo da protagonista nella costruzione dell'immagine mediatica dei migranti. Va precisato che il mese di settembre del 2015 rappresenta il culmine della crisi migratoria con i più alti numeri di arrivati nel Mediterraneo ${ }^{3}$, mentre nell'aprile del 2017, anche se i giornali continuano a parlare degli alti numeri di arrivati, come temi predominanti si presentano l'accoglienza e il salvataggio dei migranti. Al culmine della crisi migratoria del 2015 corrisponde un elevato interesse del pubblico e dei media, il che coincide con maggior numero degli articoli individuati nel settembre del 2015 rispetto al numero degli articoli dell'aprile del 2017.

\subsection{Genericizzazione}

Secondo vari studi e ricerche, tra i termini più ricorrenti nella denominazione dei migranti nel discorso mediatico italiano risultano le parole migrante, immigrato, rifugiato, profugo, straniero, richiedente asilo, mentre recentemente le parole irregolare, clandestino ed

\footnotetext{
${ }^{1}$ http://www.repubblica.it/ (pagina consultata il 30/4/2017).

2 http://www.ilgiornale.it/ (pagina consultata il 30/4/2017).

3 Secondo i dati dell'UNHCR, il numero di arrivati nel Mediterraneo risulta il più alto nel 2015: 225455 arrivi nel 2014, 1032408 arrivi nel 2015, 373652 arrivi nel 2016, 185139 arrivi nel 2017, 141472 arrivi nel 2018. https://data2.unhcr.org/en/situations/mediterranean\# (pagina consultata il 27/9/2019).
} 
extracomunitario vengono evitate per le connotazioni negative che le accompagnano (Taylor 2009, 2014, Faloppa 2011, Orrù 2017). Si tratta dei riferimenti generici che abbracciano le parole più ricorrenti nella denominazione dei migranti anche nei due corpora analizzati (Tabella 1 e Tabella 2).

\section{Tabella 1}

la Repubblica / il Giornale

14-30 settembre 2015

158 articoli (99634 parole)

\begin{tabular}{lc} 
migrante & $43,91 \%$ \\
profugo & $23,85 \%$ \\
rifugiato & $13,37 \%$ \\
immigrato & $7,38 \%$ \\
straniero & $6,22 \%$ \\
richiedente asilo & $4,20 \%$ \\
clandestino & $1,01 \%$ \\
extracomunitario & $0,04 \%$ \\
irregolare & $0,02 \%$ \\
\hline
\end{tabular}

\section{Tabella 2}

la Repubblica / il Giornale

14-30 aprile 2017

91 articoli (47273 parole)

migrante

immigrato

straniero

rifugiato

profugo

clandestino

richiedente asilo

extracomunitario

irregolare frequenza d'uso

$64,21 \%$

$9 \%$

$7,36 \%$

$5,93 \%$

$5,11 \%$

$3,07 \%$

$2,45 \%$

$1,64 \%$

$1,23 \%$

Va sottolineato che le parole citate maggiormente vengono usate al plurale, mentre la parola più frequentemente usata risulta essere la parola dal significato più generico migrante, spesso usata anche come sinonimo delle parole dal significato più ristretto quali immigrato, rifugiato, profugo, (1), (2) e (3). Questo abuso della parola migrante accenna a un disordine terminologico nonché alla noncuranza 
nei confronti delle persone che migrano e cercano la propria salvezza in Italia.

(1) Quando riescono ad arrivare a Melilla, i rifugiati vengono alloggiati nel centro di permanenza temporanea per immigrati (Ceti), un luogo "destinato a fornire servizi e prestazioni sociali di base", con 512 posti e che attualmente, secondo i dati dell'Unhcr, ospita circa 1.600 migranti, nell'80\% dei casi siriani. (la Repubblica, 26/9/2015)

(2) A quasi un anno dalla chiusura delle frontiere di Ungheria, Croazia, Bulgaria e Grecia la rotta balcanica ha lasciato oltre $74 m i l a$ profughi in completo stallo. La Serbia - unico stato a non aver chiuso i confini - ospita migliaia di migranti bloccati in improvvisati centri di accoglienza. (la Repubblica, 26/4/2017)

(3) Uno dei due centri per migranti era stato edificato, infatti, senza il "permesso di costruire", mentre i proprietari del secondo immobile avevano presentato un "falso attestato di agibilità", per essere abilitati all'accoglienza dei profughi. (il Giornale, 28/4/2017)

È da notare che nell'aprile 2017 l'incidenza della parola migrante, calcolata in relazione alle occorrenze degli altri riferimenti generici analizzati, aumenta di più del $20 \%$ rispetto all'incidenza evidenziata nel settembre 2015. Va anche notato che nel corpus del 2017 si assiste a un uso ridotto delle parole profugo e rifugiato che risultano meno ricorrenti delle parole immigrato e straniero dal significato più generico e con sfumature semantiche negative. Si può ipotizzare che un ovvio aumento dell'incidenza della parola migrante nella denominazione delle persone che migrano e un uso ridotto delle parole profugo e rifugiato siano un accenno non solo alla noncuranza nei confronti dei nuovi arrivati, ma anche a un atto di presa di distanze nato per le svariate ragioni tra le quali si annovera anche l'ignoranza (4).

(4) Perché non si possono fare distinzioni tra migranti, profughi, rifugiati e le cause che li spingono a fuggire. (la Repubblica, 25/9/2015)

Tra gli altri riferimenti generici evidenziati negli articoli analizzati, è interessante osservare l'impiego dell'aggettivo denominale $i$ disperati. Si tratta della parola relativa sia allo stato emotivo sia alle condizioni economiche dei migranti i quali, qualificati come disperati, vengono percepiti come persone senza speranza e senza possessi, e perciò come un peso, prima di tutto finanziario, per la società italiana 
(Sciortino e Colombo 2004, Orrù 2017). Negli articoli del settembre 2015 questa parola spesso viene qualificata dagli attributi quali inseguiti dalla guerra, in fuga da guerra e miseria, provenienti in gran parte della Siria, in fuga dai propri paesi, che indicano le ragioni del loro stato di disperati giustificando la loro fuga ed evocando la compassione, (5) e (6). Però, nell'aprile 2017 si assiste a una notevole riduzione dell'impiego della parola cosicché essa viene evidenziata soltanto in pochi esempi, il che si potrebbe interpretare come un'altra dimostrazione di presa di distanze.

(5) Si conta che il modello dell'accoglienza diffusa sappia anche in questo caso assorbire un numero consistente di disperati in fuga dai propri paesi. (la Repubblica, 22/9/2015)

(6) Anzi, a cinque azioni, tutte collegate a un obiettivo molto concreto: la realizzazione di un corridoio piuttosto umanitario che apra la strada dell'Europa ai disperati insequiti dalla querra in nome della dignità umana come valore fondante dell'Unione europea. (la Repubblica, 23/9/2015)

Attraverso la categoria della qualificazione i migranti vengono associati a varie sfere concettuali tra le quali predominano l'arrivo, il salvataggio e la morte (cfr. ter Wal 2001, Orrù 2017). Si tratta delle tre aree tematiche più consistenti che abbracciano il maggior numero di qualifiche in tutti e due i corpora. Però, va notato che nel settembre del 2015 gli articoli analizzati sono maggiormente incentrati sulla tematica dell'arrivo, (7) e (8), mentre negli articoli dell'aprile del 2017 l'attenzione si sposta sulle tematiche del salvataggio e della morte nel Mediterraneo, (9) e (10).

(7) Per l'agenzia per Ue per la gestione delle frontiere esterne (Frontex) nel mese di agosto sono entrati nell'Ue 156mila migranti, portando il totale da inizio anno a oltre 500mila. 11 mezzo milione di ingressi in otto mesi è già quasi il doppio rispetto ai 280mila migranti che erano arrivati nell'Ue in tutto il 2014. (Ia Repubblica, 15/9/2015)

(8) Non si allenta, comunque, la tensione tra i vari Paesi: la Croazia ha già fatto sapere che chiederà che la Grecia smetta di smistare i migranti in arrivo verso il resto dell'Ue. (la Repubblica, 21/9/2015)

(9) $\grave{E}$ bastato, infatti, I'arrivo del rimorchiatore Asso 29 con $\underline{1.800}$ immigrati a bordo, soccorsi nel canale di Sicilia nelle ultime ore, per mandare in tilt la piccola questura di Vibo Valentia. (il Giornale, 19/4/2017) 
(10)Questi nuovi decessi portano a 37 il numero di uomini, donne e bambini morti in mare sulla rotta del Mediterraneo orientale dall'inizio del 2017 e a 1.089 il numero totale di decessi nel Mediterraneo. (la Repubblica, 25/4/2017)

\subsection{Collettivizzazione}

A parlare della denominazione, è interessante osservare il riferimento alla nazionalità, notato in diversi studi sulla raffigurazione mediatica dei migranti come un accenno alla loro diversità e non appartenenza alla società italiana ed europea (Sciortino, Colombo 2004, Montali et al. 2013, Taylor 2014, Orrù 2017). Nei corpora analizzati, nei casi in cui la nazionalità viene indicata, più frequentemente si tratta dei dati statistici concernenti la presenza straniera nel territorio italiano o europeo (11), mentre il riferimento alla nazionalità ricorre anche nelle notizie sui crimini commessi da parte degli stranieri, maggiormente come l'unica informazione sui protagonisti della notizia (12).

(11) ... quando già è notte inoltrata e tra le luci del porto e delle navi cargo attraccate ai pontili sta per concludersi un altro sbarco dalla nave militare tedesca "Werra" con 140 persone, 84 maschi, 44 donne, 12 minori, quasi tutti nigeriani in fuga da Boko Haram. (Ia Repubblica, 29/9/2015)

(12) L'analisi sottolinea anche significative differenze nella propensione al crimine a seconda del paese d'origine: $i$ più pericolosi sarebbero algerini, marocchini e tunisini. Molto meno siriani e iracheni. Quasi un terzo degli immigrati giunti di recente nel paese è recidivo, sottolinea lo studio, e il cinque per cento di loro ha commesso almeno sei reati. (il Giornale, 24/4/2017)

Negli articoli del 2015 i migranti vengono distinti come senegalesi, siriani, eritrei, ghanesi, somali, afgani, nigeriani, pachistani/pakistani, egiziani, iracheni e bengalesi. D'altra parte, negli articoli del 2017 si nota un uso ridotto del riferimento alla nazionalità documentato attraverso minor numero di riferimenti (senegalesi, tunisini, marocchini, srilankesi, nigeriani, bengalesi) e di occorrenze.

Oltre a essere collettivizzati in base alla nazionalità, i migranti vengono anche percepiti come un'entità attraverso l'uso dei riferimenti collettivi esseri umani, massa umana, folla, persone e gente. Spesso i nomi gente e persone vengono accompagnati dagli aggettivi disperato, stravolto, spaventato, bisognoso e dalle espressioni in fuga, senza terra 
i quali alludono a una situazione difficile condivisa da tutti i migranti in cerca di un futuro migliore nei paesi europei, (13) e (14).

(13)I volontari austriaci, ungheresi e tedeschi che correvano incontro al flusso di gente stravolta, a quelli che avevano marciato lungo l'autostrada, a quelli che approdavano con $i$ treni e i bus. (la Repubblica, 15/9/2015)

(14)E, come spiega anche la Stampa, il bel tempo dei giorni scorsi ha spinto persone disperate ad attraversare il Mar Mediterraneo nel tentativo di raggiungere I'Italia e da qui arrivare nei Paesi del Nord Europa. (il Giornale, 17/4/2017)

\subsection{Quantificazione}

Molto spesso i migranti vengono enumerati, classificati e presentati numericamente (Montali et al. 2013, Orrù 2017). La quantificazione, come tra l'altro l'impiego dei riferimenti generici e il frequente riferimento alla nazionalità, contribuisce alla percezione dei migranti come una moltitudine non differenziata, una folla priva d'identità, a una sorta di categorizzazione, ovvero a un processo di spersonalizzazione o di personalizzazione senza la persona (RFC 2002, Dal Lago 1999). I migranti e i rifugiati vengono trattati come numeri, con toni spesso allarmanti particolarmente presenti nelle espressioni generiche e indefinite, come ad esempio centinaia di migranti, migliaia di migranti, centinaia di migliaia di migranti, troppi migranti. Ricorrono spesso i riferimenti al numero degli arrivi, degli sbarchi, dei salvati oppure dei morti nel Mediterraneo, dei migranti, dei profughi, dei rifugiati, dei richiedenti asilo, dei disperati, degli uomini, delle donne e dei bambini stranieri ecc., (15), (16) e (17).

(15) Nei primi cinque mesi del 2015, più di 42mila persone, per lo più rifugiati, ha raggiunto la Grecia via mare, secondo I'Unhcr. L'agenzia dell'Onu per i rifugiati ha detto che più di 300mila persone finora hanno rischiato la vita per attraversare il mar Mediterraneo quest'anno, con circa 2.500 rifugiati e migranti morti o dispersi nel tentativo di raggiungere l'Europa. (la Repubblica, 27/9/2015)

(16) Dopo le decine di soccorsi nel Mediterraneo di imbarcazioni colme di migranti, $i$ due giorni più di 4000 persone, iniziano gli sbarchi nei porti siciliani. Stamattina alle 7,30 è approdata al porto di Catania la nave tedesca Tender A513 Rhein con a bordo circa 1100 migranti salvati nel Canale di Sicilia. (la Repubblica, 17/4/2017) 
(17) Nonostante il peggioramento dalla scorsa notte delle condizioni meteorologiche, migliaia di immigrati non hanno rinviato la propria partenza dalla Libia: duemila sono sbarcati venerdi, 4.500 sabato e altri 200 ieri. (il Giornale, 17/4/2017)

\subsection{Sintagmi tra accoglienza e diffidenza}

Dato che i fenomeni migratori figurano tra i più dirompenti che popolano le cronache italiane, studiando gli articoli giornalistici la prima impressione che si impone riguarda una serie di sintagmi con cui si descrive il fenomeno. Accanto ai riferimenti generici e collettivi i termini che accompagnano l'immigrato e l'immigrazione alimentano la diffidenza e innalzano le barriere perché si tratta di una serie di parole che presentano l'immigrazione prima di tutto come un pericolo sociale (ter Wal 2001, ter Wall 2002, Orrù 2017). Si osserva quindi una certa oscillazione dei due periodi trattati e analizzati ossia quello del 2015 e 2017.

In un contesto di allarme crescente, cioè nel periodo culmine che si riferisce al 2015, si nota la forte presenza dei sintagmi costruiti intorno ai lemmi crisi, dramma, problema, gestione ed emergenza. Si parla così della crisi dei rifugiati, crisi dei profughi, crisi migratoria, del dramma dei profughi, dramma dei migranti, dramma dell'immigrazione, del problema dei migranti, problema dei profughi, problema dei flussi migratori, della gestione della crisi migranti, gestione del flusso dei migranti, gestione dell'immigrazione, dell'emergenza dell'immigrazione, emergenza migranti, (18), (19), (20), (21) e (22).

(18) "La politica europea deve essere più attenta ai bisogni, più attenta al sociale", ha detto la Boldrini, riferendosi sempre al problema della immigrazione e all'atteggiamento che dovrebbe assumere l'Ue su questo fenomeno. (il Giornale, 14/9/2015)

(19) Il capo dello Stato Mattarella il 16 settembre ha qualificato l'invasione come "un fenomeno epocale (...) che richiede una gestione comune dell'Unione". (il Giornale, 18/9/2015)

(20) Solo risposte comuni europee possono fronteggiare il dramma dell'immigrazione. Sarà questa la linea che il presidente italiano Sergio Mattarella porterà oggi nella sua prima visita a Vienna. (la Repubblica, 16/9/2015)

(21) I capi di Stato e di governo dell'Unione europea cercheranno di mandare un segnale di unità e responsabilità incontrandosi oggi a Bruxelles per affrontare l'emergenza migranti. (la Repubblica, 23/9/2015) 
(22) Prima ancora di suscitare tensioni in Europa, la crisi dei migranti ha prosciugato le finanze delle agenzie dell'Onu e delle Ong che col conflitto siriano hanno ormai raggiunto il limite del possibile. (la Repubblica, 28/9/2015)

In altre parole l'immigrazione crea l'imbarazzo sociale, porta alla crisi e diventa un problema da risolvere (Montali et al. 2013, Orrù 2017). Inoltre il repertorio di forme linguistiche che si aggiunge nella trattazione dei giornali italiani tende ad associare il problema dell'immigrazione al lemma questione e alle formulazioni come la questione migratoria, la questione dei siriani, la questione dei flussi migratori, la questione dei profughi e dei migranti. Siccome ogni questione richiede una soluzione si cerca la risposta che non viene trovata e che diventa un vero peso per la società, da una parte diffidente e intollerante e dall'altra accogliente e sensibile al fenomeno, (23). Nel processo dell'accoglienza molto spesso si parla della registrazione dei migranti, della distribuzione dei migranti, della redistribuzione dei migranti, del ricollocamento dei migranti e del rimpatrio dei migranti, (24) e (25).

(23) Il presidente del Consiglio ha parlato del problema dei profughi e della questione siriana, ricordando il ruolo centrale dell'Italia "la prima a cogliere" I'importanza della crisi dei migranti, iniziata nel Mediterraneo. (la Repubblica, 29/9/2015)

(24) Mentre I'Unione europea continua a litigare sul piano di ricollocamento dei migranti (Hollande ha detto che "nessun Paese si può esimere") in attesa del Consiglio Europeo di mercoledi, dall'altra parte dell'Oceano gli Stati Uniti confermano le loro aperture sull'accoglienza. (Ia Repubblica, 20/9/2015)

(25) Tra le decisioni anche quella di aprire i centri di identificazione in Italia e in Grecia e avviare la ridistribuzione e $i$ rimpatri dei migranti entro novembre. (la Repubblica, 25/9/2015)

Quando nel 2017 i toni allarmistici leggermente si attenuano, la crisi migratoria si trasforma nel fenomeno migratorio, spiccano i lemmi dell'accoglienza e del salvataggio, del traffico e dei trafficanti. Tali formulazioni risultano con il fenomeno migratorio, fenomeno dell'immigrazione; l'accoglienza dei migranti, l'accoglienza dei profughi, il business dell'accoglienza, l'accoglienza al collasso; il salvataggio dei migranti, le Ong salvamigranti; il traffico dei migranti, i trafficanti dei migranti, il traffico di esseri umani, i trafficanti di esseri umani, (26), (27), (28), (29) e (30). 
(26) C'è un nostro disegno preciso per fare fronte ad un tema che, come detto, non è più un'emergenza. Non dobbiamo quindi più seguire il fenomeno immigrazione ma governarlo e dobbiamo mettere meno inquietudine nelle persone'. (la Repubblica, 21/4/2017)

(27) Sull'argomento sono intervenute oggi le dirette interessate, ovvero le organizzazioni impegnate con le proprie navi nel salvatagaio dei migranti del Mar Mediterraneo. (il Giornale, 24/4/2017)

(28) Sulla vicenda è intervenuto il senatore di Forza Italia, Renato Schifani, che ha parlato di "business dell'accoglienza". (il Giornale, 28/4/2017)

(29) Significativo lo smarcamento da Zuccaro del collega Francesco Lo Voi, procuratore capo di Palermo che pure indaga sul traffico di migranti. (la Repubblica, 28/4/2017)

(30) Ovvero, rilasciando dichiarazioni quotidiane ai media nazionali sulla presunta collusione tra alcune ong e i trafficanti di esseri umani, fino ad adombrare il sospetto che le organizzazioni criminali libiche arrivino a finanziare alcune sigle umanitarie per finalità eversive. (la Repubblica, 28/4/2017)

Altri modi con cui si qualificano i protagonisti del fenomeno migratorio fanno riferimento alle condizioni disperate in cui si trovano, ma anche a una serie di limitazioni relative all'ingresso e alla permanenza e infine alla loro effettiva integrazione. Così si incontrano le costruzioni in cui si parla dei migranti che occupano il posto, che devastano, protestano, minacciano (31), e mentre da una parte si proclama l'accoglienza incondizionata dei profughi, la difesa dei profughi, la solidarietà con i profughi (32), dall'altra si parla della protesta dei profughi che sono falsi e arroganti e vanno rimpatriati.

(31)A Trapani la scuola materna Don Bosco è stata occupata nei giorni scorsi da un gruppo di migranti che protestavano per le condizioni di accoglienza dell'Italia. (il Giornale, 17/9/2015)

(32)Restano ammassati attorno alla piccola stazione della ferrovia, diventata ormai il simbolo di un esodo che nessuno immaginava cosi intenso e senza fine. I piccoli gesti di solidarietà, la compostezza, perfino i silenzi sono quasi una forma di rispetto nei confronti di chi ha perso tutto. (Ia Repubblica, 20/9/2015) 


\section{Conclusione}

Pur essendoci le frontiere mentali, nelle società europee in generale, la nuova realtà creatasi con le nuove migrazioni, ha fatto nascere la consapevolezza della coabitazione, cioè della significativa presenza delle persone percepite come oggettivamente diverse e con cui convivere. $\mathrm{Ci}$ si trova di fronte a uno strano fenomeno di "personalizzazione senza la persona" trattandosi delle persone che non hanno bagagli e non portano documenti, non hanno né nome né identità, sradicate dalla loro terra, dalla loro lingua e dalla loro quotidianità.

Nonostante vari studi e progetti volti ad analizzare il ruolo della lingua nel discorso mediatico e a evidenziare l'importanza di un corretto uso della lingua, nella denominazione dei protagonisti del fenomeno migratorio è ancora presente un disordine terminologico. Inoltre, essi vengono raffigurati come una folla priva d'identità attraverso l'impiego di diverse strategie discorsive, il che contribuisce alla costruzione di una percezione negativa spesso accrescendo l'allarmismo e il sensazionalismo.

Nel presente studio si è cercato di osservare le differenze nella raffigurazione dei migranti trattati negli articoli giornalistici italiani, pubblicati nei due periodi corrispondenti ai differenti contesti sociopolitici della realtà italiana. Si evidenzia una lieve differenza nell'uso di riferimenti generici, riferimenti collettivi e qualificatori che riguardano i due corpora. Si può constatare che nel corpus del 2017, a differenza del corpus del 2015 centrato sulla tematica dell'arrivo percepito come minaccia alla società europea, gli insiemi di parole rappresentano le sfere concettuali dell'accoglienza e della convivenza mettendo in risalto, da una parte la condizione del migrare e della drammaticità del viaggio e dall'altra il problema dell'inserimento, di superflui quantificatori e qualificatori di nazionalità o provenienza che possono risultare con atteggiamenti discriminatori e di ostilità. L'immigrato viene raffigurato soprattutto attraverso la descrizione delle caratteristiche etniche o il riferimento al Paese di provenienza e in tal modo considerato più come rappresentante di una categoria di persone che come una persona singola. A questo proposito, occorre innanzitutto non ignorare il loro stato di invisibilità civile, non etichettarli e non dare spazio ai sensazionalismi e agli stereotipi dell'appartenenza etnica.

Considerata l'importanza dei media nella formazione dell'opinione pubblica, vogliamo sperare che questo intervento possa contribuire a rendere il discorso mediatico meno allarmistico e discriminatorio, 
rispettoso della diversità, e che aiuti a far "comunicare senza discriminare" ${ }^{4}$.

\section{Bibliografia:}

Dal Lago, Alessandro. Non-persone. L'esclusione dei migranti in una società globale. Milano: Feltrinelli, 2009 [1999].

Faloppa, Federico. Lessico e alterità, la formulazione del "Diverso". Alessandria: Edizioni dell'Orso, 2000.

Parole contro. La rappresentazione del "diverso" nella lingua italiana e nei dialetti. Torino: Garzanti, 2004. Razzisti a parole (per tacer dei fatti). Roma-Bari: Laterza, 2011.

Maneri, Marcello. "Lo straniero consensuale. La devianza degli immigrati come circolarità di pratiche e discorsi". In II nemico e lo straniero: materiali per l'etnografia contemporanea. Ed. Dal Lago, Alessandro. Genova: Costa \& Nolan, 1998. 236-272.

Montali, Lorenzo et al. "The Representation of Migrants in the Italian Press. A Study on the Corriere della Sera (1992-2009)". Journal of Language and Politics 12.2 (2013): 226-50.

Orrù, Paolo. Il discorso sulle migrazioni nell'Italia contemporanea. Un'analisi linguistico-discorsiva sulla stampa (2000-2010). Milano: Franco Angeli, 2017.

Reisigl, Martin e Ruth Wodak. Discourse and Discrimination. Rhetorics of Racism and Antisemitism. London: Routledge, 2001.

RFC. Rapporto finale della Fondazione Censis. L'immagine degli immigrati e delle minoranze etniche nei media. Roma: Fondazione Censis, 2002.

Sciortino, Giuseppe e Asher Colombo. "The flows and the flood: the public discourse on immigration in Italy, 1969-2001". Journal of Modern Italian Studies, 9.1 (2004): 94-113.

Taylor, Charlotte. The representation of immigrants in the Italian press. CIRCaP, Occasional Papers 21 (2009). Siena: Università di Siena. "Investigating the representation of migrants in the UK and Italian press". International Journal of Corpus Linguistics, 19.3 (2014): 368-400.

\footnotetext{
4 "Comunicare senza discriminare" è il motto del progetto Parlare civile e dell'omonimo sito web ideati con lo scopo di "fornire un aiuto pratico a giornalisti e comunicatori per trattare con linguaggio corretto temi sensibili e a rischio di discriminazione". http://www.parlarecivile.it/home.aspx (pagina consultata l'11/10/2019).
} 
Ter Wal, Jessica. "Comparing Argumentation and CounterArgumentation in Italian Parliamentary Debate on Immigration". In The Semiotics of Racism. Ed. Wodak Ruth. Wien: Passagen Verlag, 2000. 129-154.

"Minacce territoriali, socio-economiche e di sicurezza. L'immagine degli immigrati nella stampa quotidiana". Incontri 16 (2001): 67-78.

Ter Wal, Jessica. (a cura di). "Italy". In Racism and Cultural Diversity in the Mass Media. An Overview of Research and Examples of Good Practice in the 15 EU Member States, Vienna: European Monitoring Centre on Racism and Xenophobia, 2002. 239-272.

Triandafyllidou, Anna. "Nation and Immigration: A Study of the Italian Press Discourse". Social Identities, 5.1(1999): 65-88.

\section{Sitografia}

http://www.repubblica.it/. 30 aprile 2017.

http://www.ilgiornale.it/. 30 aprile 2017.

https://data2.unhcr.org/. 27 settembre 2019.

http://www.parlarecivile.it/. 11 ottobre 2019.

\section{MEDIJSKI PRIKAZ MIGRANATA IZMEĐU PRIHVAĆANJA I NEPOVJERENJA}

Posljednjih desetljeća dvadesetog stoljeća novi ratovi, etničko čišćenje i ekološke katastrofe doveli su do povećanja broja migranata i izbjeglica, ljudi u bijegu i u tranzitu koji se sele u potrazi za boljim životnim uvjetima. Europske zemlje, posebno Italija, pogođene sindromom invazije zbog kontinuiranog dolaska imigranata, osjećaju se u opasnosti. Stoga su podignuti novi zidovi, i to ne samo oni stvarni, nego i mentalni zidovi i granice. Nova klima pogodovala je nastanku novih riječi koje se odnose na migracije i poimanje migranata. $S$ porastom fenomena migracija proširila se epidemija predrasuda i stereotipa prema ljudima percipiranima kao objektivno različitima. Među riječima i izrazima koji se koriste pri imenovanju i opisivanju novih došljaka učestalo je etiketiranje, što pridonosi formiranju uglavnom negativnog medijskog prikaza migranata.

Različite studije koje se bave ovom temom pokazuju da talijanski medijski diskurs, usredotočen na alarmantnost, doprinosi stvaranju negativnih stereotipa o strancima, povezanima $s$ kriminalom i opasnošću. Prema Maneriju i Dal Lagu, stranca se doživljava kao prijetnju talijanskom i europskom društvu te diskriminira, u prvom 
redu, pomoću jezika kojim ga se prikazuje, dok se opozicija Mi/Oni pojačava generalizacijama i upotrebom metaforičkog leksika.

Korpus analiziran u ovom radu obuhvaća članke o temi migracija, preuzete iz dviju talijanskih dnevnih novina. $S$ ciljem definiranja uloge jezika u konstrukciji medijske slike o migrantima, proučavaju se članci objavljeni tijekom dva razdoblja (rujan 2015. i travanj 2017.) koja odgovaraju različitim društveno-političkim kontekstima talijanske stvarnosti.

Ključne riječi: fenomen migracija, talijanski medijski diskurs, diskursne strategije, negativni stereotipi o strancima, prihvaćanje i nepovjerenje 